\title{
Small nucleolar RNA 78 promotes the tumorigenesis in non-small cell lung cancer
}

\author{
Di Zheng ${ }^{1 *}$, Jie Zhang ${ }^{2}$, Jian Ni ${ }^{1}$, Jie Luo ${ }^{1}$, Jiying Wang ${ }^{1}$, Liang Tang ${ }^{2}$, Ling Zhang ${ }^{1}$, Li Wang ${ }^{1}$, Jianfang X ${ }^{1}$, \\ Bo Su ${ }^{2}$ and Gang Chen ${ }^{3^{*}}$
}

\begin{abstract}
Background: Accumulating evidence suggests that dysregulated snoRNA may play a role in the development of malignancy. In the present study, we investigated the role of SNORD78 in the tumorigenesis of non-small cell lung cancer (NSCLC).

Methods: We determined the expression level of SNORD78 in NSCLC tissues with quantitative real-time PCR and then studied its clinical significance. We explored the biological significance of SNORD78 with gain-and-loss-of-function analyses both in vitro and in vivo.

Results: A great upregulation of SNORD78 was observed in cancer tissues compared to their adjacent normal tissues. Meanwhile, patients with high SNORD78 expression have significantly poorer prognosis than those with low expression. Inhibition of SNORD78 suppressed the proliferation of NSCLC cells via inducing G0/G1 cell cycle arrest and apoptosis while SNORD78 overexpression promoted the cell proliferation. SNORD78 promoted invasion of NSCLC cells via inducing epithelial-mesenchymal-transition (EMT). SNORD78 was also obviously upregulated in cancer stem-like cells and is required for the self-renewal of NSCLC. The oncogenic activity of SNORD78 was also confirmed with in vivo data.
\end{abstract}

Conclusion: Our study identified that SNORD78 may be a potential therapeutic target for NSCLC.

Keywords: Non-small cell lung cancer, Small nucleolar RNA, SNORD78, Epithelial-mesenchymal-transition, Cancer stem-like cell

\section{Introduction}

Lung cancer represents the most frequent cause of cancer-related mortality [1]. Non-small cell lung cancer (NSCLC), including adenocarcinoma and squamous cell carcinoma, accounts for about $85 \%$ of all lung cancers [2]. Most of NSCLC patients are diagnosed at advanced stages due to the fact that they are usually asymptomatic at early stages [3]. In spite of the recent advances in the management of NSCLC, the prognosis of NSCLC remains relatively poor [4]. Therefore, it is of urgent need to identify novel biomarker of NSCLC and reveal the precise molecular mechanism of the development of NSCLC.

\footnotetext{
*Correspondence: zhengdiok@aliyun.com; chestpathology@126.com 'Department of Medical Oncology, Shanghai Pulmonary Hospital, Tongji University School of Medicine, 507 Zhengmin Road, Shanghai 200433, People's Republic of China

${ }^{3}$ Department of Pathology, Zhongshan Hospital, Fudan University, 180 Fenglin Road, Shanghai 200032, People's Republic of China Full list of author information is available at the end of the article
}

Recently, accumulating literature has demonstrated that noncoding RNAs (ncRNAs), including microRNAs (miRNAs), long noncoding RNAs (lncRNAs) and small nucleolar RNAs (snoRNAs), play an important role in the pathogenesis of NSCLC [5-7]. Small nucleolar RNAs are a group of molecules range between 60-300 nucleotides (nt) in length and represent one of the best characterized groups of ncRNAs [8]. They are prominently found in the nucleolus and functions to guide RNAs for post-transcriptional modification of ribosomal RNAs and some spliceosomal RNAs [9]. From a structural basis, snoRNAs fall into two categories termed box C/D snoRNAs (SNORDs) and box H/ACA snoRNAs (SNORAs) $[9,10]$. SNORDs define the target sites for 2'-O-ribose methylation of rRNAs or snRNAs, whereas SNORAs define the target sites for the isomerization of uridine residues into pseudouridine [10]. A growing volume of literature has indicated that dysregulated snoRNA may play a role in the development of malignancy. For example, Mei 
et al. [7] demonstrated that SNORA42 acts an oncogene in lung cancer. SNORD33, SNORD66, SNORD73B, SNOR D76, SNORD78, and SNORA42 could be potential biomarker of non-small-cell lung cancer [11]. SNORD113-1 functions as a tumor suppressor role in hepatocellular carcinoma [9].

A growing volume of work has proposed that the cancer cell population are heterogeneous, and that a relatively small subpopulation of cancer cells often termed "cancer stem-like cells" (CSCs) or "tumor-initiating cells" harbored enhanced therapy resistance and brisk tumorigenicity $[12,13]$. As a result, CSCs are responsible for tumor propagation, resistance to conventional therapy, and tumor recurrence [14]. Mannoor et al. [15] profiled snoRNA expression in cancer stem cells of lung cancer and found that several snoRNAs were significantly upregulated in tumor-initiating cells of lung cancer. We found that snoRD78 was consistently reported to be upregulated in lung cancer [11] and tumor-initiating cells of lung cancer [15], suggesting that SNORD78 might play a role in lung tumorigenesis.

However, the role of SNORD78 in cancer is elusive and few studies have examined its molecular mechanism in NSCLC. In this study, we would like to explore the role of SNORD78 in NSCLC.

\section{Materials and methods \\ Cell culture}

Three NSCLC adenocarcinoma cell lines (A549, SPCA1, NCI-H1975), a NSCLC squamous carcinomas cell line (SK-MES-1), and a normal human bronchial epithelial cell line (16HBE) were purchased from the Institute of Biochemistry and Cell Biology of the Chinese Academy of Sciences (Shanghai, China) and were authenticated. The cell lines were cultured in DMEM or RPMI 1640 (Gibco $\mathrm{BRL}$ ), containing $10 \%$ fetal bovine serum (FBS, HyClone) as well as $100 \mathrm{U} / \mathrm{ml}$ penicillin and $100 \mu \mathrm{g} / \mathrm{ml}$ streptomycin (Invitrogen). Cells were maintained in a humidified incubator at $37{ }^{\circ} \mathrm{C}$ in the presence of $5 \% \mathrm{CO}_{2}$. All cell lines have been passaged for fewer than 6 months.

\section{Patient and clinical samples}

The human specimens in this study were sanctioned by the local ethics committee of Pulmonary Hospital at Tongji University (Shanghai, China). A total of 56 primary cancer tissues and paired adjacent noncancerous tissues ( $>5 \mathrm{~cm}$ from tumor) were obtained from patients who underwent surgery at Pulmonary Hospital between 2008 and 2011 and were diagnosed with NSCLC (stage II, III, and IV) based on histopathological evaluation. These tissues were flash frozen in liquid nitrogen immediately after surgery and subsequently stored at $-80{ }^{\circ} \mathrm{C}$ prior to RNA isolation and qRT-PCR analysis. No patients received anticancer treatments before surgery in this study. Complete clinicopathological data of the patients from which the specimens were collected were available. Overall survival (OS) was defined as the interval between the dates of surgery and death.

\section{RNA extraction and quantitative real-time PCR}

Total RNA was isolated from tumor tissues and purchased cell lines using TRIzol (Life Technologies) according to supplier's instructions. RNA concentration was measured with a NanoDrop ND-2000 spectrophotometer (Life Technologies). Reverse transcription was performed with random primers using the First Strand cDNA synthesis kit (Takara, Otsu, Shiga, Japan) according to the manufacturer's instructions. The quantitative real-time polymerase chain reaction (qRT-PCR) was performed using the SYBR Select Master Mix (Applied Biosystems, cat: 4472908) on ABI 7500 system (Applied Biosystems, CA, USA) according to the manufacturer's instructions. $\beta$-actin was measured as an internal control. The relative expression fold change of mRNAs was calculated by the $2^{-\Delta \Delta C t}$ method. After the reverse transcription, $0.5 \mu \mathrm{l}$ of the complementary DNA was used for subsequent qRT-PCR reaction. The primer sequences were as follows: $\beta$-actin: 5-GAAATCGTGCGTGACA TTAA-3' (forward), 5'-AAGGAAGGCTGGAAGAGTG-3' (reverse); GAS5: 5'-TGAAGTCCTAAAGAGCAAGCC-3' (forward), 5'-ACCAGGAGCAGAACCATTAAG-3' (reverse); SNORD78: 5'-GTGTAATGATGTTGATCAAATG TCTGAC-3' (forward), 5'-CACATTACTACAACTAGTT TACAGACTGG-3' (reverse).

For cell expression and tumor samples, each sample was run in triplicate. qRT-PCR results were analyzed and expressed relative to CT (threshold cycle) values, and then converted to fold changes.

\section{Plasmid, shRNA and cell transfection}

Small interfering RNAs targeting GAS5 were bought from Genepharm Technologies (Shanghai, China). Expression vectors encoding SNORD78 were purchased from Fulen Gen Company (Guangzhou China). For stable knockdown of SNORD78, NSCLC cells were transfected with lentiviral constructs encoding two different SNORD78 shRNAs or nontargeting shRNAs in the presence of polybrene $(8 \mathrm{mg} / \mathrm{mL})$. After $48 \mathrm{~h}$, transduced cells were grown in culture media containing $1 \mathrm{mg} / \mathrm{mL}$ puromycin for two weeks. The shRNA sequences were as the followings: shRNA1: GTTGATCAAATGTCTGACCTG, shRNA2: GACCTGAAATGAGCATGTAGA. For construction of lentiviral vector expressing human SNORD78 gene, SNORD78 cDNA was amplificated by PCR with the primers (forward, 5'-ggggtaccGTGTAATGAT-3', and reverse, 5'-ccgctcgagTGTAGACAAAGGTA-3'). To obtain cell lines stably expressing SNORD78, A549 cells were infected with the LV-SNORD78 and LV-control viruses. The 
stable over-expressing cell lines were identified using realtime PCR and named as A549 LV-SNORD78 clone 1 and LV-SNORD78 clone2.

All transfected cells were selected with puromycin $(1 \mathrm{mg} / \mathrm{ml})$ for two weeks.

\section{Cancer cell invasion assay}

Cancer cell invasion assay was performed using Boyden chambers with filter inserts (pore size, $8.0 \mu \mathrm{m}$, Millipore, MA) coated with Matrigel (40 $\mu$ l, Sigma, USA) in 24-well dishes (Corning). Briefly, $1-2 \times 10^{5}$ cells after transfected with SNORD78 shRNA or control shRNA, LV-SNORD78 or LV-control were seeded in the upper chamber, while the culture medium (Invitrogen) with $10 \%$ FBS was placed in the lower chamber. The plates were incubated for $24 \mathrm{~h}$ or $48 \mathrm{~h}$. The cells on the upper surface were scraped and washed away, whereas the cells on the lower surface were fixed in $4 \%$ formaldehyde and stained with $0.05 \%$ crystal violet for $2 \mathrm{~h}$. Finally, cells were counted under a microscope and the relative number was calculated. Experiments were independently repeated in triplicate.

\section{Cell viability assay}

Cell viability was assessed by the Cell Counting Kit 8 (CCK-8, Donjindo). Briefly, control and treated A549 and H1975 cells were seeded into 96-well plates at an initial density of 3000 and 5000 cells/well. At each time points, $10 \mu \mathrm{l}$ of CCK-8 solution was added to each well and incubated for $2 \mathrm{~h}$. The absorbance was measured by scanning with a microplate reader at $450 \mathrm{~nm}$. Data were expressed as the percentage of viable cells as follows: relative viability $(\%)=\left(\mathrm{A} 450_{\text {treated }}-\mathrm{A} 450_{\text {blank }}\right) /$ $\left(\mathrm{A} 450_{\text {control }}-\mathrm{A} 450_{\text {blank }}\right) \times 100 \%$.

\section{Cell cycle analysis}

Cells transiently transfected with desired vector were harvested $48 \mathrm{~h}$ after transfection. The cells were washed for three times by cold PBS, and then cells were fixed in $70 \%$ ethanol in PBS at $-20{ }^{\circ} \mathrm{C}$ for $24 \mathrm{~h}$. After fixation, cells were washed with cold PBS and stained with $0.5 \mathrm{ml}$ of propidium iodide (PI) staining buffer, which contains $200 \mathrm{mg} / \mathrm{ml}$ RNase A, $50 \mu \mathrm{g} / \mathrm{ml} \mathrm{PI}$, at $37{ }^{\circ} \mathrm{C}$ for $30 \mathrm{~min}$ at $4{ }^{\circ} \mathrm{C}$ in the dark. The cell-cycle profiles were assayed at $488 \mathrm{~nm}$ on an EPICS 752 flow cytometer (Coulter, Hialeah, FL) equipped with MPLUS software (Phoenix 140 Flow Systems, San Diego, CA). Data were expressed as percentage distribution of cells in G0/G1, S and G2/M phases of the cell cycle.

\section{Western blot analysis}

The harvested cells were centrifuged at 2,000 rpm for $5 \mathrm{~min}$. The total cellular proteins were prepared using RIPA cell lysis buffer (Cell Signaling Technology) supplemented with protease inhibitors. The lysates were then collected and subjected to ultrasonication and centrifugation. The supernatants were collected, and protein content was determined by Bradford assay. Equal amounts $(30-50 \mu \mathrm{g})$ of proteins were applied to an 8$12 \%$ SDS-polyacrylamide separating gel and transferred to a PVDF Immobilon-P membrane (Millipore). The membrane was blocked with $5 \%$ nonfat milk in TBST and then probed with indicated primary antibodies with gentle shaking at $4{ }^{\circ} \mathrm{C}$ overnight. The membranes were washed with TBST $(3 \times 10 \mathrm{~min})$, incubated in secondary antibodies at room temperature for $1 \mathrm{~h}$. Antibody-bound proteins were detected by BeyoECL Plus kit.

The primary antibodies used in these experiments include E-cadherin (1:1000) (Abcam), N-cadherin (1:1000) (Abcam), Vimentin (1:500) (Abcam), p21 (1:1000), p16 (1:1000) and caspase-3 (1:1000). HRP-conjugated goat anti-rabbit IgG antibody (Abcam) was used as the secondary antibody.

\section{Immunofluorescence analysis}

Cells transfected with desired vector were cultured and fixed on $12 \times 12 \mathrm{~mm}$ glass slides. For membrane staining (E-cadherin), cells were fixed with cold $100 \%$ methanol for $10 \mathrm{~min}$. For intracellular staining (Vimentin, Ncadherin), the cells were fixed with $4 \%$ (wt/vol) paraformaldehyde in PBS and permeabilized by incubation with $0.5 \%$ Triton X-100 in PBS for 2 min. The cells were incubated with $5 \%$ bovine serum albumin in PBS for $20 \mathrm{~min}$ at room temperature. After washing with PBS, the cells were incubated with specific primary antibody at $4{ }^{\circ} \mathrm{C}$ overnight. Antibody dilutions of 1:400 were used for E-cadherin (Abcam), N-cadherin (Abcam) and Vimentin (Cell Signaling Technology). The cells were then washed and incubated with Alexa Fluor 488conjugated goat anti-rabbit IgG for $1 \mathrm{~h}$. The nuclei were stained with 4,6-diamidino-2-phenylindole (DAPI). Sections were visualized by a LEICA DMI 3000B fluorescence microscope with an original magnification $\times 200$.

\section{Mammosphere-formation assay}

Single cells were plated in ultralow-attachment six-well plate (Corning) at a density of 10,000 viable cells $/ \mathrm{mL}$. Cells were grown in a serum-free DMEM/F12 medium (Invitrogen), supplemented with $20 \mathrm{ng} / \mathrm{mL}$ EGF and $20 \mathrm{ng} / \mathrm{mL}$ bFGF (BD Biosciences). Bovine pituitary extract was excluded. Fresh medium were added $0.5 \mathrm{ml} /$ well every three days. Mammospheres $>50 \mu \mathrm{m}$ in diameter were counted at day 8 . Images were taken with a LEICA DMI 3000B with original magnification $\times 100$ or $\times 400$. 


\section{Methylation-specific polymerase chain reaction}

The methylation status of the E-cadherin promoter region was determined by methylation-specific PCR (MSP) using bisulfite-modified DNA. Genomic DNA was extracted using the QIAamp DNA mini kit (Axygen). Two primer sets were used to amplify the promoter region of the E-cadherin that incorporated a number of $\mathrm{CpG}$ sites, one specific for the methylated sequence (E-cadherin- $M$, forward: 5'-TATGAGTTGTAAGCGGTAGAGTTC-3'; reverse: 5'-TACGAACTTAACGAAAAAAAATCAT-3') and the other for the unmethylated sequence (E-cadherin- $\mathrm{U}$, forward: 5'- GAATATGAGTTGTAAGTG GTAGAGTT T-3'; reverse: 5'-TACAAACTTAACAAAAAAAAATCA TACT-3'). The primers used in the present study detect specifically the promoter sequence of the E-cadherin. $\mathrm{M}$ and $\mathrm{U}$ are the PCR products of methylated and unmethylated alleles, respectively. The polymerase chain reactions for E-cadherin-M and E-cadherin- $\mathrm{U}$ were carried out in a $50 \mu \mathrm{l}$ volume containing $1 \times$ polymerase chain reaction buffer $\left(15 \mathrm{mmol} / \mathrm{l} \mathrm{MgCl}_{2}\right), 2.5 \mathrm{mmol} / \mathrm{l}$ mixture of dNTPs, 10 pMof each primer, 4 U HotStart Taq DNApolymerase (Qiagen, Frankfurt, Germany), and $25 \mathrm{ng}$ to $50 \mathrm{ng}$ of bisulfitemodified DNA. Amplification was performed in a thermocycler with the following conditions: $94{ }^{\circ} \mathrm{C}$ for $3 \mathrm{~min}$, cycled at $94{ }^{\circ} \mathrm{C}$ for $15 \mathrm{~s}, 60{ }^{\circ} \mathrm{C}$ or $57{ }^{\circ} \mathrm{C}$ for $15 \mathrm{~s}$, and $72{ }^{\circ} \mathrm{C}$ for $15 \mathrm{~s}$ ( 35 cycles), followed by an extension at $72{ }^{\circ} \mathrm{C}$ for $7 \mathrm{~min}$. Methylation-specific PCR experiments were performed at least in duplicate.

\section{Animal experiments}

Briefly, male BALB/cathymic nude mice (4-6 weeks old) were obtained from the experimental animal center of Shanghai Institute for Biological Science. To evaluate the effect of SNORD78 in nude mice, we constructed NSCLC H1975 cells with SNORD78 stable down expression. All mice were injected subcutaneously into either side of flank area with $1 \times 10^{7}$ SNORD78 stablely downregulated or control $\mathrm{H} 1975$ cells to establish the NSCLC xenograft model. Tumor volumes were measured $(0.5 \times$ length $\times$ width $^{2}$ ) in mice on a weekly basis. After 6 weeks, mice were sacrificed, and tumors were exercised and subjected to immunohistochemical analysis of Ki67. All animal experiments were performed in animal laboratory center of Pulmonary Hospital and in accordance with the Guide for the Care and Use of Laboratory Animals published by the US National Institutes of Health (NIH publication no. 85-23, revised 1996). The study protocol

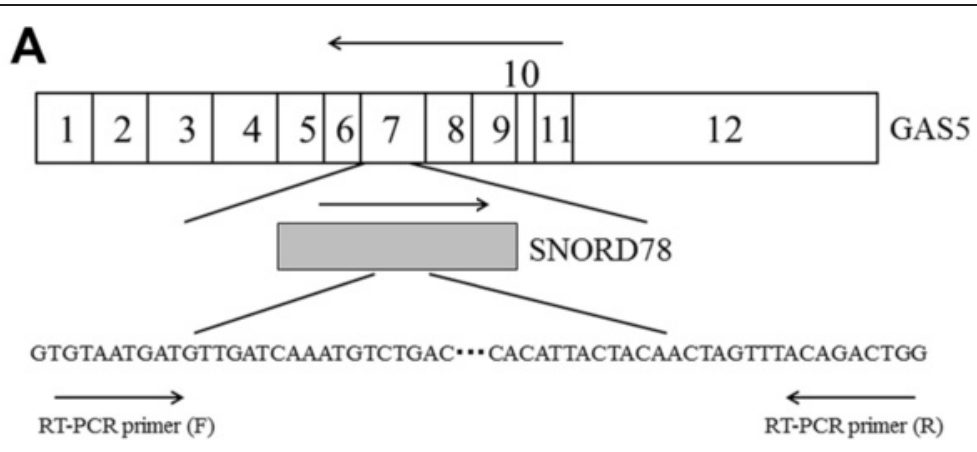

B
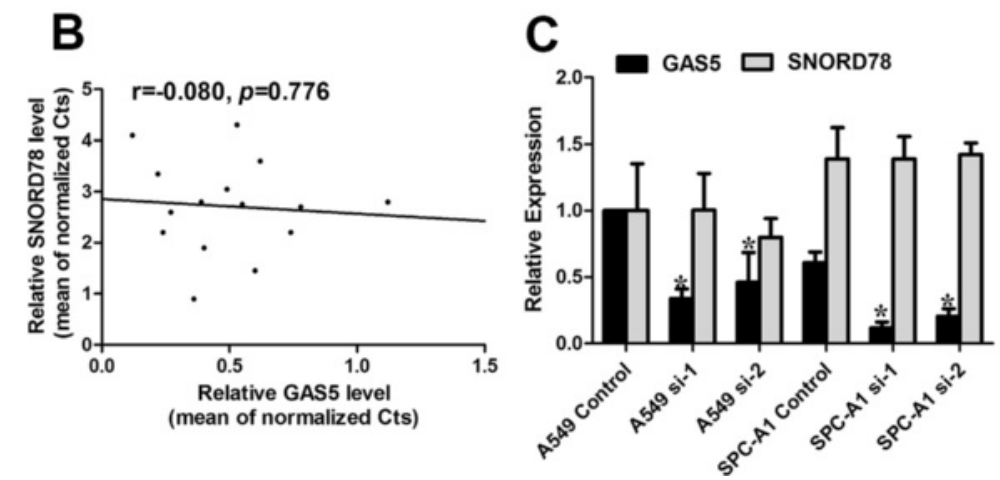

Fig. 1 SNORD78 is transcribed independently of GAS5. (a) Schematic diagram of SNORD78 and its location in GAS5. GAS5 (top) consists of 12 exons and SNORD78 resides in intron 7-8 of GAS5. SNORD78 is depicted as the gray box. The location of the forward (F) and reverse (R) primers used for RT-PCR are shown. Orientation of arrows indicates the direction of the transcription or amplification reaction. (b) Linear correlation between the expression of the SNORD78 and GAS5 was not observed. $\triangle \mathrm{Ct}$ values were used to measure gene expression, which was normalized by $\beta$-actin expression levels. (c) Expression of GAS5 was decreased, and SNORD78 was not significantly changed in A549 and SPC-A1 cells with two different siRNAs against GAS5. Statistical differences were analyzed relative to siRNA control. *, $p<0.05$ 
was approved by the Animal Care and Use committee of Pulmonary Hospital.

\section{Statistical analysis}

All statistical analyses were performed with SPSS version 19.0 software (SPSS software, Armonk, NY, USA). All data were presented as mean \pm standard deviation from three independent experiments with each measured in triplicate. The gene expression level of SNORD78 in tumors was compared with adjacent normal tissues utilizing the paired sample $t$-test, whereas the association between SNORD78 expression and clinical characteristics was evaluated using the chi-square test. Survival curves were plotted by the Kaplan-Meier method, and the long-rank comparison was carried out to assess differences between stratified survival groups using the

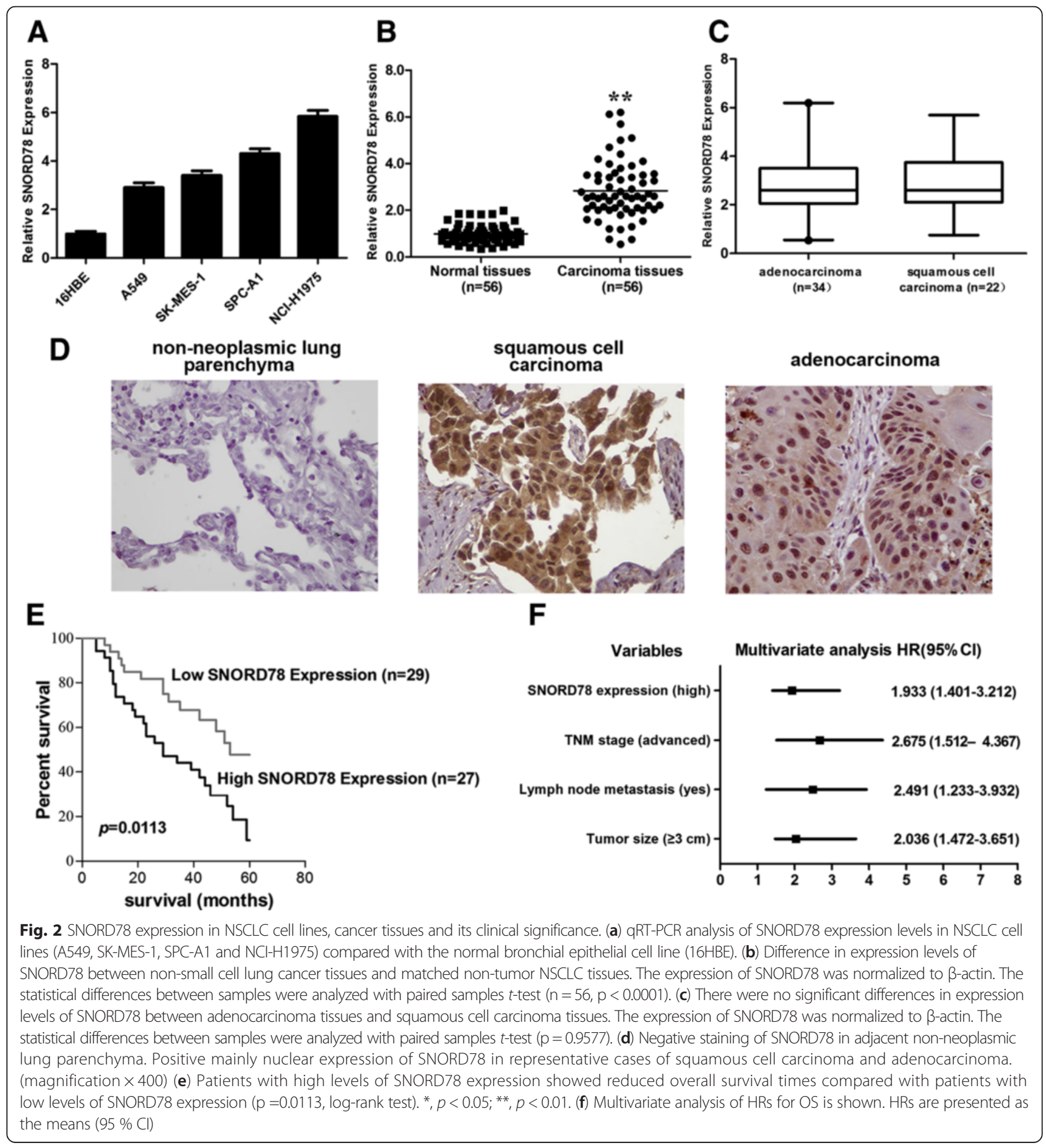


Table 1 Correlation between SNORD78 expression and clinicopathologic characteristics

\begin{tabular}{|c|c|c|c|c|}
\hline \multirow[b]{2}{*}{ Clinicopathologic parameters } & \multicolumn{2}{|c|}{$\begin{array}{l}\text { SNORD78 } \\
\text { expression }\end{array}$} & \multirow[b]{2}{*}{ Number of cases } & \multirow[b]{2}{*}{$p$ value } \\
\hline & High & Low & & \\
\hline Age (years) & 10 & 14 & 24 & 0.2301 \\
\hline$\leq 60$ & 14 & 18 & 32 & \\
\hline \multicolumn{5}{|l|}{$>60$} \\
\hline \multicolumn{5}{|l|}{ Gender } \\
\hline Male & 13 & 11 & 24 & 0.4043 \\
\hline Female & 13 & 19 & 32 & \\
\hline \multicolumn{5}{|l|}{ Diameter } \\
\hline$\leq 3$ & 11 & 9 & 20 & $0.024^{\mathrm{a}}$ \\
\hline$>3$ & 24 & 12 & 36 & \\
\hline \multicolumn{5}{|l|}{ Lymph node metastasis } \\
\hline Yes & 21 & 9 & 30 & $0.0004^{a}$ \\
\hline No & 13 & 13 & 26 & \\
\hline \multicolumn{5}{|l|}{ TNM stage } \\
\hline$|-| \mid$ & 5 & 16 & 21 & $0.0354^{a}$ \\
\hline III & 25 & 10 & 35 & \\
\hline \multicolumn{5}{|l|}{ Degree of differentiation } \\
\hline Well and moderately & 14 & 16 & 30 & 0.3778 \\
\hline Poorly & 15 & 11 & 26 & \\
\hline
\end{tabular}

${ }^{a}$ Statistically significant $(p<0.05)$

median value as the cutoff. A Cox proportional hazards analysis was performed to calculate the hazard ratio (HR) and the $95 \%$ confidence interval (CI) to evaluate the association between SNORD78 expression and survival. Cox regression analysis was used to determine the independent factors, which were based on the variables selected by univariate analysis. The expression differences between cell lines, the expression changes after transfection, cell viability and cell invasion assays were analyzed using independent samples $t$-test.

\section{Results}

Characterization of SNORD78 in NSCLC

SNORD78 is located in 1q25, one of most frequently amplified chromosomal segments in solid tumors, particularly NSCLC [16-18]. Furthermore, we performed a computational screen and found that SNORD78 resides in intron 7-8 of GAS5 (Fig. 1a). To explore the potential relationship between the SNORD78 and GAS5 transcripts, we first examined the expression levels of SNORD78 and GAS5 in 15 NSCLC tissues. The results showed that no correlation $(\mathrm{r}=-0.080, p=0.776)$ existed between the transcriptional levels of SNORD78 and GAS5 (Fig. 1b). Furthermore, SNORD78 was statistically unchanged in A549 and SPC-A1 cells transfected with two different short interfering (siRNAs) (designated as si-1 and si-2) against GAS5, despite significant reduction in GAS5 messenger RNA expression (Fig. 1c).

\section{SNORD78 was upregulated in NSCLC}

SNORD78 was reported to be upregulated in lung cancer tissues [11]. To further corroborate the previous finding of Liao et al. [11], we firstly detected SNORD78 expression in a series of lung cancer cell lines including adenocarcinoma and squamous carcinoma subtypes utilizing qRT-PCR. When normalized to normal bronchial epithelial cell line (16HBE), the RNA level of SNORD78 was increased in the NSCLC cancer cell lines (A549, SKMES-1, SPC-A1, NCI-H1975) by 2.7- to 5.8-fold compared with 16HBE (Fig. 2a).

We detected SNORD78 expression in 56 pairs of NSCLC tissues and in their adjacent normal tissues utilizing qRT-PCR. The data showed that SNORD78 expression was obviously increased, and the average intensity was increased 3-fold in tumorous than in adjacent normal tissues (Fig. 2b). The average $\Delta \mathrm{Ct}$ expression value of SNORD78 was 6.75 in NSCLC tissues, ranking from 5.91 to 8.32 , when compared with $\beta$-actin. Furthermore, there were no statistical differences of expression of SNORD78 between adenocarcinoma and squamous cell

Table 2 Univariate and multivariate analysis of factors associated with overall surivival in NSCLC patients (56 pairs)

\begin{tabular}{|c|c|c|c|c|}
\hline \multirow[b]{2}{*}{ Risk factors } & \multicolumn{2}{|l|}{ Univariate analysis } & \multicolumn{2}{|l|}{ Multivariate analysis } \\
\hline & $\mathrm{HR}(95 \% \mathrm{Cl})$ & $p$ value & $\mathrm{HR}(95 \% \mathrm{Cl})$ & $p$ value \\
\hline $\operatorname{Age}(\leq 60 />60)$ & $2.899(0.997-8.426)$ & 0.063 & & \\
\hline Gender(male/female) & $3.618(0.948-13.812)$ & 0.06 & & \\
\hline Diameter $(\geq 3 /<3)$ & $3.125(1.093-8.929)$ & $0.035^{\mathrm{a}}$ & $2.036(1.472-3.651)$ & $0.011^{\mathrm{a}}$ \\
\hline Lymph node metastasis(Yes/No) & $3.703(1.119-11.507)$ & $0.014^{\mathrm{a}}$ & $2.491(1.233-3.932)$ & $0.015^{\mathrm{a}}$ \\
\hline TNM stage (III/I-II) & $4.689(1.633-14.506)$ & $0.030^{\mathrm{a}}$ & $2.675(1.512-4.367)$ & $0.001^{\mathrm{a}}$ \\
\hline SNORD78 expression(high/low) & $5.495(1.678-17.857)$ & $0.005^{\mathrm{a}}$ & $1.933(1.401-3.212)$ & $0.007^{\mathrm{a}}$ \\
\hline Histological grade (poorly/well and moderately) & $1.666(0.652-4.259)$ & 0.408 & & \\
\hline
\end{tabular}

${ }^{\text {a }}$ tatistically significant $(p<0.05)$ 


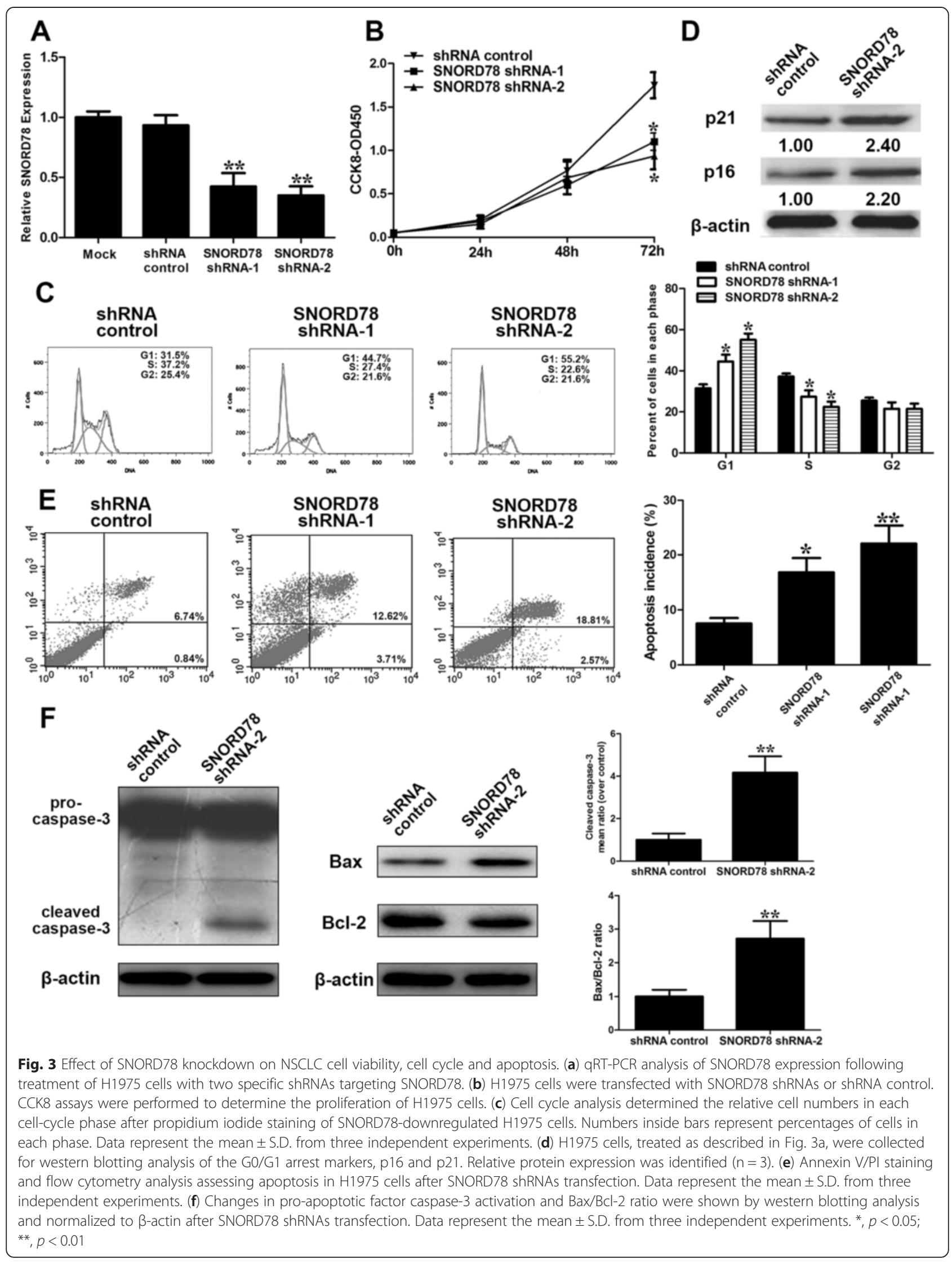


carcinoma, suggesting that SNORD78 was shared in two major histological types of NSCLC (Fig. 2c). In situ hybridization (ISH) studies confirmed the upregulation of SNORD78 in NSCLC (Fig. 2d).

\section{The association between SNORD78, clinicopathological} characteristics and prognosis after surgery

The patients with NSCLC were then stratified according to SNORD78 expression level (median split) and compared for different clinicopathologic features (age, sex, tumor size, lymph node status, distant metastasis, and survival time). Results revealed that SNORD78 expression was positively correlated with the TNM stage $(p=0.0354$, $p<0.05)$, lymph node metastasis $(p=0.0004, p<0.05)$ and tumor size $(p=0.024, p<0.05)$. There was no significant correlation between SNORD78 expression with age, gender, or degree of differentiation (Table 1).

Kaplan-Meier survival analysis and log-rank tests using patient postoperative survival were conducted to further evaluate the correlation between SNORD78 and prognosis of patients with NSCLC. The cumulative survival rate was significantly better in patients with lower-SNORD78- expressing tumours than in those with higher- SNORD78expressing tumors $(p=0.0113$, HR $(95 \%$ CI $)=1.933$ (1.401-3.212)); Fig. 2d). In addition, the univariate analysis revealed that the tumor size, lymph node metastasis, TNM stage and SNORD78 expression were significantly correlated with overall survival (Table 2). The multivariate analysis demonstrated that tumor size $(p=0.011)$, lymph node metastasis $(p=0.015)$, TNM stage $(p=$ $0.001)$ and SNORD78 expression $(p=0.007)$ were independent risk factors for overall survival (Fig. 2e) (Table 2).

\section{SNORD78 is required for efficient proliferation of NSCLC cells}

The fact that SNORD78 is frequently upregulated in primary NSCLC tissues and NSCLC cells prompted us to explore the role of SNORD78 dysregulation in NSCLC cells. We constructed cell lines that stably downregulated SNORD78 by lentivirus infection of H1975 cells with specific shRNAs (Fig. 3a), which expressed relatively higher level of SNORD78. The CCK-8-based assay detected significant decrease in the proliferation of

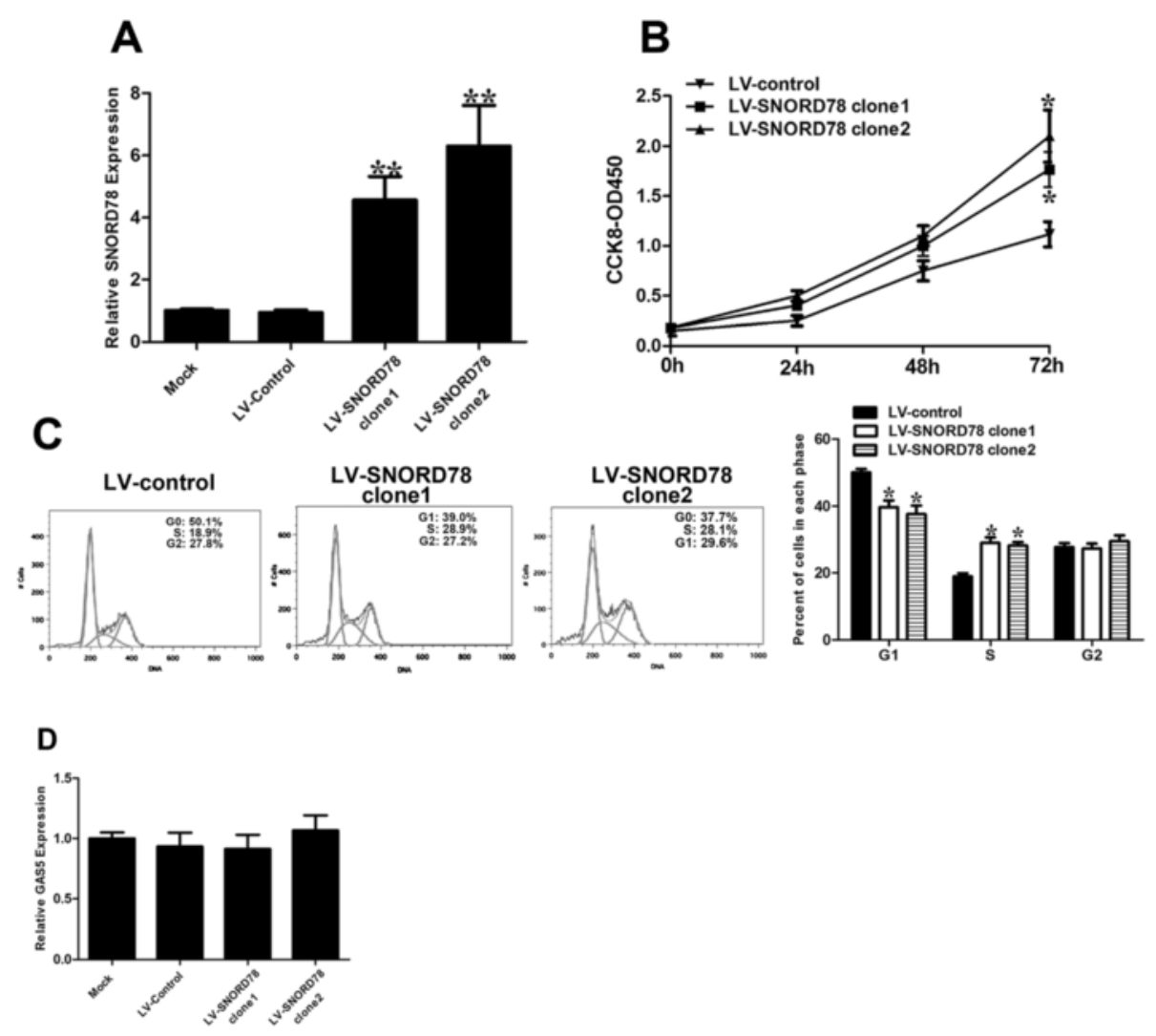

Fig. 4 Effect of SNORD78 overexpression on NSCLC cell viability and cell cycle. (a) qRT-PCR analysis of SNORD78 expression following transfection of A549 cells with SNORD78. (b) A549 cells were transfected with SNORD78 or control. CCK8 assays were performed to determine the proliferation of A549 cells. (c) Cell cycle analysis determined the relative cell numbers in each cell-cycle phase after propidium iodide staining of SNORD78 overexpressed A549 cells. Numbers inside bars represent percentages of cells in each phase. (d) qRT-PCR analysis of GAS5 expression following transfection of A549 cells with SNORD78. Data represent the mean \pm S.D. from three independent experiments. ${ }^{*}, p<0.05 ;{ }^{* *}, p<0.01$ 


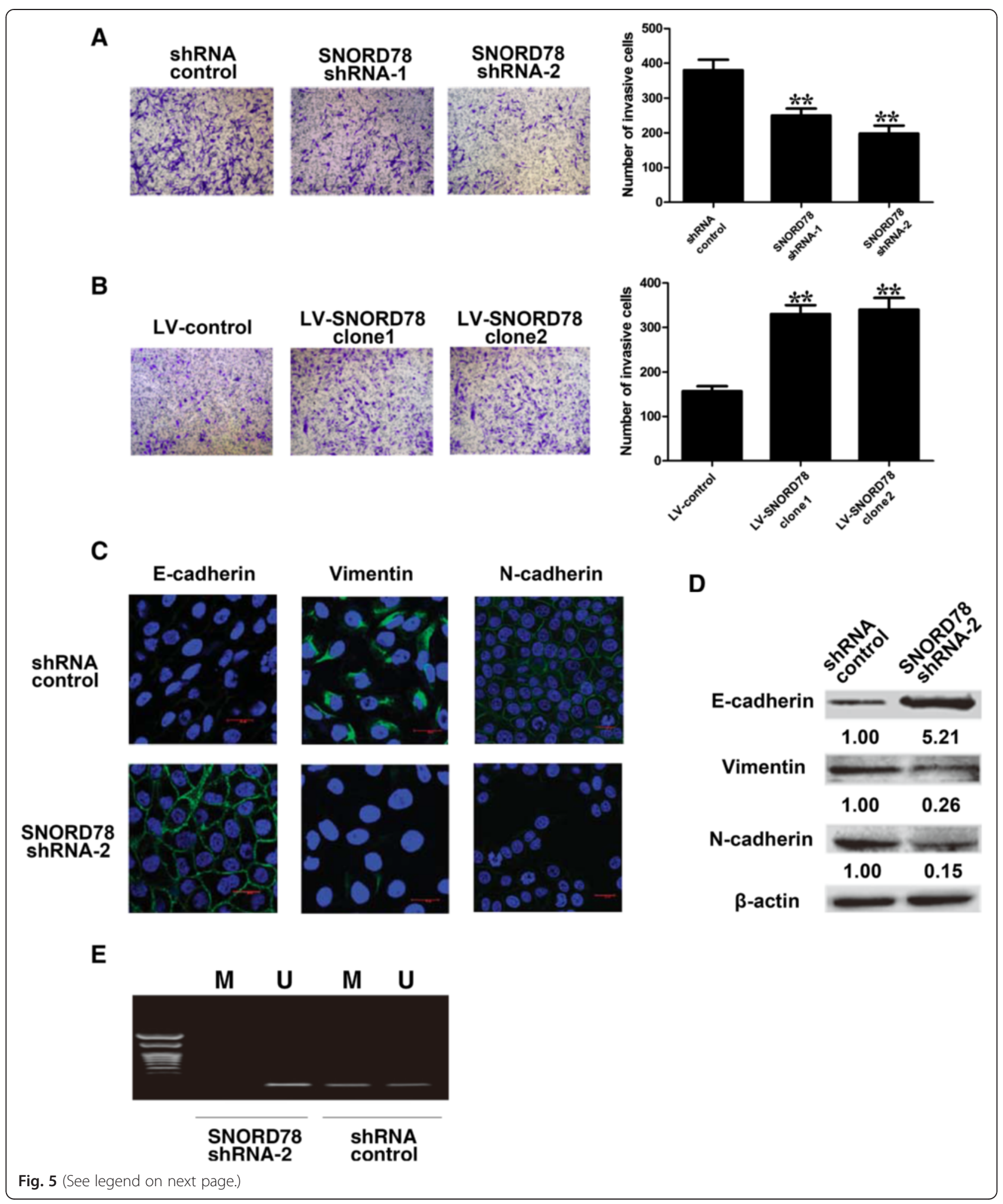


(See figure on previous page.)

Fig. 5 SNORD78 promoted invasion of NSCLC cells via inducing epithelial-mesenchymal-transition (EMT). (a) H1975 cells were transfected with shRNA control or shRNA SNORD78. Transwell assays were performed to investigate the invasive ability of H1975 cells. Data represent the mean \pm S.D. from three independent experiments. (b) A549 cells were transfected with LV-control or LV-SNORD78. Transwell assays were performed to investigate the invasive ability of A549 cells. Data represent the mean \pm S.D. from three independent experiments. ${ }^{*}, p<0.05 ;{ }^{* *}, p<0.01$. (c) immunofluorescence images of H1975 cells stained using antibodies against E-cadherin, Vimentin or N-cadherin after transfected with shRNA control or SNORD78 shRNA-2 (original magnification $\times 200$ ). (d) Western-blot analysis of phenotypic markers after SNORD78 knockdown in H1975 cells. Relative protein expression was identified $(n=3)$ and normalized to $\beta$-actin. Data represent the mean \pm S.D.. (e) Results of MSP analysis of E-cadherin gene in SNORD78 shRNA-2-transfected H1975 cells, and control cells. M and U, PCR products of methylated and unmethylated alleles, respectively

H1975 cells after knockdown of SNORD78 (Fig. 3b). Thus, we evaluated the effect of SNORD78 expression on cell cycle distribution and cell apoptosis in H1975 cells by flow cytometry. We found that SNORD78 silencing induced a significant G0/G1 arrest (Fig. 3c). Furthermore, the western blot analysis indicated that knockdown of SNORD78 significantly increased the expression of G0/ G1 arrest markers, p16 and p21 (Fig. 3d). These data demonstrated that SNORD78 silencing induced H1975 cell cycling arrest at the G0/G1 phase. Flow cytometry assay indicated that suppression of SNORD78 induced the increase of cell apoptosis by phycoerythrin (PE)-conjugated Annexin V staining and FACS (Fig. 3e). To explore the underlying mechanism of SNORD78's role in cell apoptosis, we determined the expression of pro-apoptotic factor, $\mathrm{Bax}, \mathrm{Bcl}$ and caspase-3 by western blot. SNORD78 knockdown increased the expression levels of cleaved caspase-3 and Bax/Bcl-2 ratio (Fig. 3f). These results demonstrated that SNORD78 knockdown may inhibit the proliferation of NSCLC cells through inducing cell G0/G1 arrest and apoptosis.

To confirm the effect of ectopic expression of SNORD78 on cell proliferation, we overexpressed SNORD78 by lentivirus infection (Fig. 4a). The results showed that the overexpression of SNORD78 increased proliferation of NSCLC cancer cell in vitro (Fig. 4b,c). To exclude the possibility that biological effects of SNORD78 depend on its regulation of GAS5, we determined the expression level of GAS5 with SNORD78 overexpression. We found that overexpression of SNORD78 had no significant effects on the expression level of GAS5 (Fig. 4d). These finding suggest that SNORD78 may take a part in the development of NSCLC.

\section{SNORD78 promoted invasion of NSCLC cells via inducing epithelial-mesenchymal-transition (EMT)}

Transwell assay revealed a substantial decrease in the number of cells that penetrated the porous filter with SNORD78 knockdown, suggesting impaired invasion ability of H1975 cells (Fig. 5a). Meanwhile, a significant increase in cancer cell invasion was observed in NSCLC cells A549 in vitro with SNORD78 overexpression (Fig. 5b). These data suggest that SNORD78 promoted the invasion of NSCLC cells. Invasion is an important characteristic of
NSCLC and emerging evidence has linked invasion with EMT. The epithelial-mesenchymal-transition (EMT) is a well-coordinated process that occurs during embryonic development and a pathological feature in tumorigenesis [19, 20]. During such a process, the epithelial phenotype cells lose the expression of E-cadherin and other components of cell to cell junctions and adopt a mesenchymal phenotype [21]. The EMT process has been shown to play a vital role in cancer invasion, metastasis, expansion of the population of cancer stem cells and therapeutic resistance [21]. We then examined the effect of SNORD78 on the EMT process of NSCLC cells.

Compared with the vector-transfected cells, H1975 cells became more round-shaped and came up with an epithelial phenotype with SNORD78 knockdown (Fig. 5c). With the immunofluorescence analysis, inhibition of SNORD78 in H1975 cells resulted in an obvious upregulation in the expression of epithelial marker E-cadherin and a great reduction in the expression of mesenchymal markers $\mathrm{N}$ cadherin and Vimentin (Fig. 5c). The western blot analysis confirmed the results from the immunofluorescence analysis (Fig. 5d). Together, it indicates that SNORD78 promoted the EMT process and thus, SNORD78 might play a role in the development of NSCLC.

As SNORDs are known to define the target sites for 2'-O-ribose methylation of rRNAs or snRNAs, we explored whether DNA methylation contributes to the EMT process with Methylation-Specific PCR to examine the role of methylation in deregulation of E-cadherin. We found that the promoter regions of E-cadherin gene from control H1975 cells were strongly methylated, whereas the SNORD78 silenced H1975 cells had unmethylated E-cadherin promoter region (Fig. 5e). These results indicate that aberrant methylation of Ecadherin gene promoter by SNORD78 may contribute to the EMT process in NSCLC.

\section{SNORD78 is required for the self-renewal of cancer-stem cells of NSCLC}

Mannoor et al. [15] profiling results revealed SNORD78 was upregulated in cancer-stem cells of NSCLC. We used $\mathrm{CD} 133^{+}$and $\mathrm{CD}_{133^{-}}$cells that were isolated from A549 cells (Fig. 6a) and confirmed that SNORD78 was specifically upregulated in cancer-stem cells (Fig. 6b). 


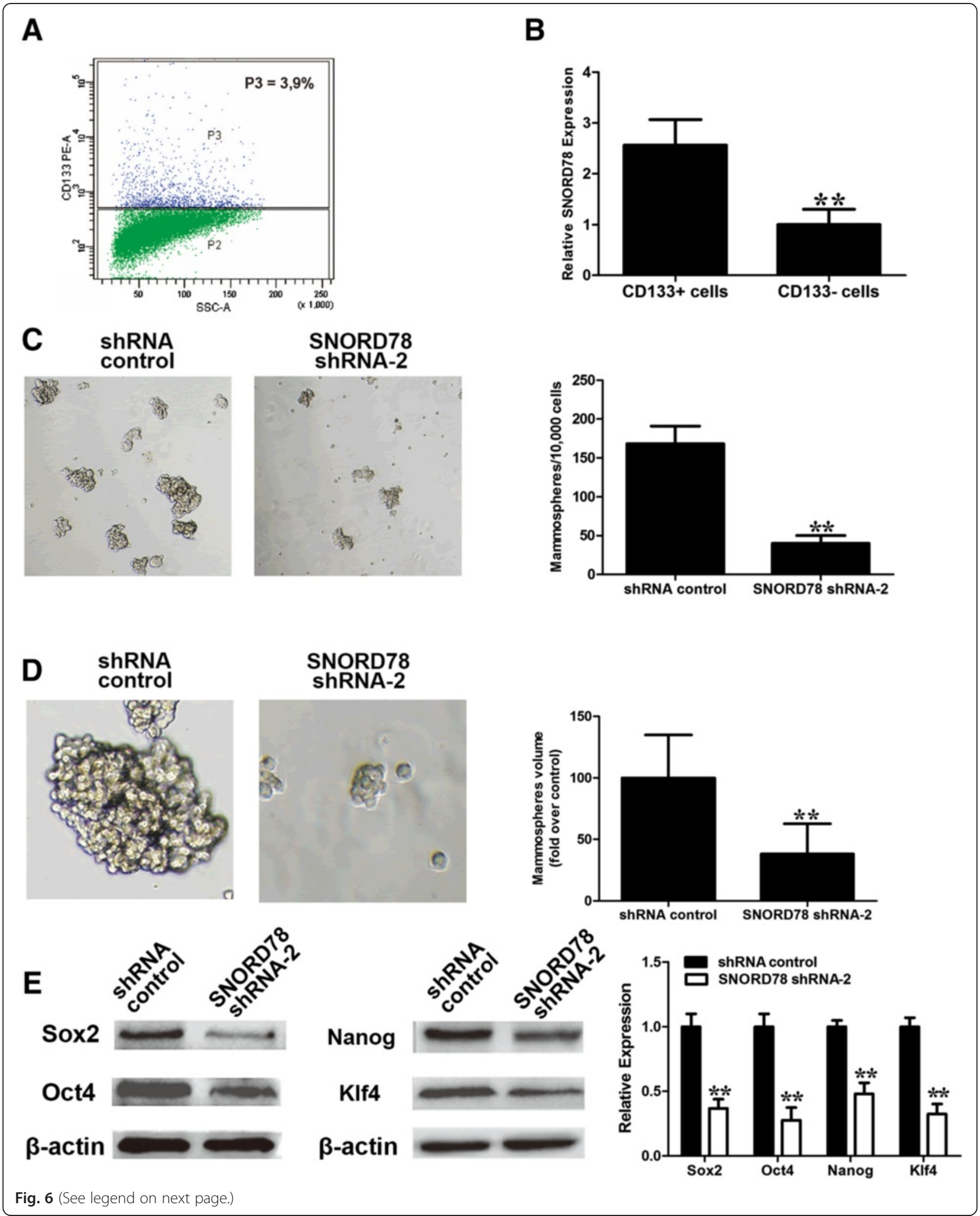


(See figure on previous page.)

Fig. 6 SNORD78 is required for the self-renewal of cancer-stem cells of NSCLC. (a) CD133 positive cells were isolated from NSCLC cells A549. (b) There was a high level of expression of SNORD78 in CD133+ cells versus CD133- cells of A549 cells as determined by qPCR analysis. (c) Representative images of mammospheres formed from A549 cells. Inhibition of SNORD78 reduced the number of mammospheres (original magnification $\times 100$ ). (d) Smaller sizes of mammospheres were observed after SNORD78 knockdown in comparison with control (original magnification $\times 400$ ). Representative images of mammospheres were shown above. Experiments were independently repeated at least three times. (e) Western-blot analysis of stemness factors after SNORD78 knockdown in H1975 cells. Relative protein expression was identified $(n=3)$ and normalized to $\beta$-actin. Data represent the mean \pm S.D..*, $p<0.05 ;{ }^{* *}, p<0.01$

Furthermore, shRNA-SNORD78 transfected cells formed fewer (Fig. 6c) and smaller mammospheres (Fig. 6d) compared with vector-transfected cells, implying that SNORD78 is required for the self-renewal of cancer-stem cells of NSCLC. Furthermore, inhibition of SNORD78 resulted in the downregulation of a series of stemness factors, which has been shown to play an important in the self-renewal of cancer stem-like cells in NSCLC $[22,23]$.

\section{SNORD78 knockdown inhibits in vivo tumorigenesis of NSCLC cells}

To validate the effect of SNORD78 on NSCLC cell tumorigenesis in vivo, H1975-shRNA-SNORD78 or H1975shRNA-control cells were injected into flanks of nude mice. Palpable tumors formed with 1 week. Tumor volume was measured on a weekly basis. Four weeks after injection, the average tumor volume of H1975 cells transfected with shRNA-SNORD78 was $1.35 \pm 0.34 \mathrm{~cm}^{3}$, which was significantly lower than tumors in the control group $\left(2.51 \pm 0.48 \mathrm{~cm}^{3}, p<0.01\right.$; Fig. 7a,b). Immunohistochemical staining of tumor tissues indicated a decrease in ki67 and an increase in cleaved caspase-3 in shRNA-SNORD78 vs. shRNA-control (Fig. 7c). The in vivo data complement the in vitro studies of SNORD78 and confirm the oncogenic activity of SNORD78 in NSCLC.

\section{Predictive value of SNORD78}

Previous reports presented that both miRNA and lncRNA can act as biomarkers for predicting progression and prognosis. In this study, we were curious about the translation of SNORD in clinical life. Therefore, we further determined the predictive potency of SNORD78 as a biomarker for metastasis. Receiver operating characteristics (ROC) curve was used to evaluate the predictive efficacy of SNORD78. For lymph node metastasis, SNORD78 alone showed low-predictive efficiency since

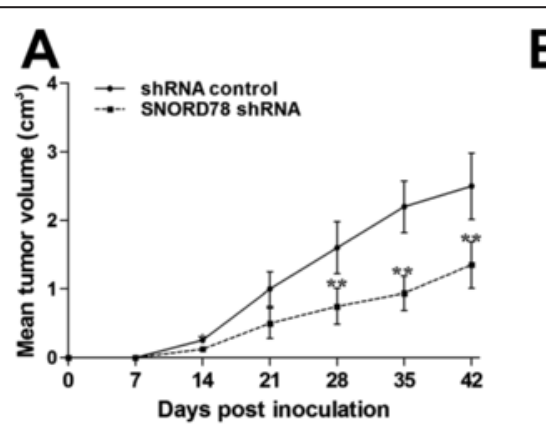

B

C

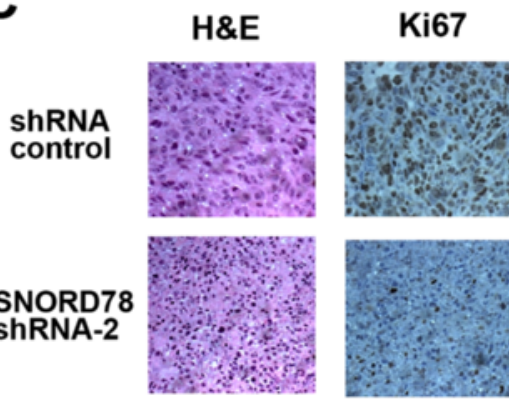

ShRNA control

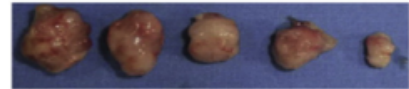

SNORD78

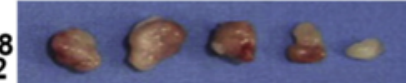

cleaved
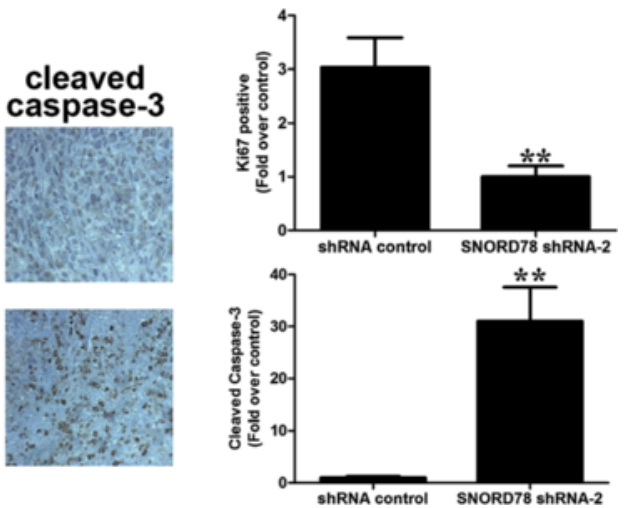

Fig. 7 The effects of SNORD78 on in vivo tumor growth of NSCLC. Inhibition of SNORD78 suppressed tumor growth in subcutaneous implantation mouse models of H1975 cells. Tumor growth curves (a) and tumor volumes (b) of subcutaneous implantation models of gallbladder cancer are shown. (c) H\&E and immunohistochemical staining demonstrated that suppression of SNORD78 inhibited the aggressive phenotype of NSCLC cells in vivo, as indicated by the expression of Ki67-positive and caspase-3-positive cells. ${ }^{*}, p<0.05 ;{ }^{* *}, p<0.01$ 
the area under the ROC curve (AUC) was 0.551 (95\% CI 0.372-0.740, $p=0.291$, Fig. 8). Next, we explored whether serum tumor biomarkers could improve the predictive efficiency of SNORD78. Among the serum biomarkers analyzed (CEA, AFP, CA125, CA19-9, and NSE), the combination of SNORD78 and CEA significantly improved the predictive efficiency (AUC $=0.642,95 \%$ CI 0.484-0.791, $\mathrm{p}=0.029$, Fig. 8).

\section{Discussion}

NSCLC is one the most frequent causes of cancerrelated motility. The understanding of NSCLC pathogenesis has improved through the identification of activating mutations in and amplifications of oncogenes, including KRAS [24], EGFR [25], KARS [26], and inactivating mutations in tumor suppressive genes, such as p53 [27, 28]. However, the mechanism of NSCLC development remains largely unknown. A growing volume of literature has indicated the role of small nucleolar RNAs in cancer $[7,9,11]$. snoRNA U50 has been demonstrated to act as a tumorsuppressor in cancer [29,30]. Mei et al. [7] reported that a diverse number of snoRNAs are differentially expressed in lung cancer with respect to the corresponding matched tissue. Mannoor et al. [15] profiled snoRNA expression in cancer stem cells of lung cancer and found that a number of snoRNAs were significantly upregulated in tumor- initiating cells of lung cancer. SNORD78 was consistently reported to be upregulated in lung cancer [11] and tumorinitiating cells of lung cancer [15], suggesting that SNORD78 might play a role in lung tumorigenesis.

In the present study, we confirmed that the expression of SNORD78 was obviously upregulated in NSCLC tissues compared with adjacent normal tissues and in cancer stem-like cells of NSCLC compared to their nonstem counterparts. Furthermore, SNORD78 was shared in two major histological types of NSCLC. SNORD78 expression was positively correlated with the TNM stage, lymph node metastasis and tumor size. High expression of SNORD78 was also associated with poor prognosis of NSCLC.

We then determine in vitro functional significance of SNORD78 in lung cancer cell lines through gain- and lossof-function analyses. We demonstrated that SNORD78 is required for efficient proliferation and invasion of NSCLC cells. Our data revealed that SNORD78 silencing inhibited cell proliferation via inducing a significant G0/G1 arrest and cell apoptosis. The proliferation-promoting effect of SNORD78 was confirmed with SNORD78 overexpression in A549 cells. SNORD78 silencing suppressed cell invasion via reversing the epithelial-mesenchymal-transition of NSCLC. The concept of cancer stem-like cells or tumorinitiating cells have proposed that the heterogeneous tumor

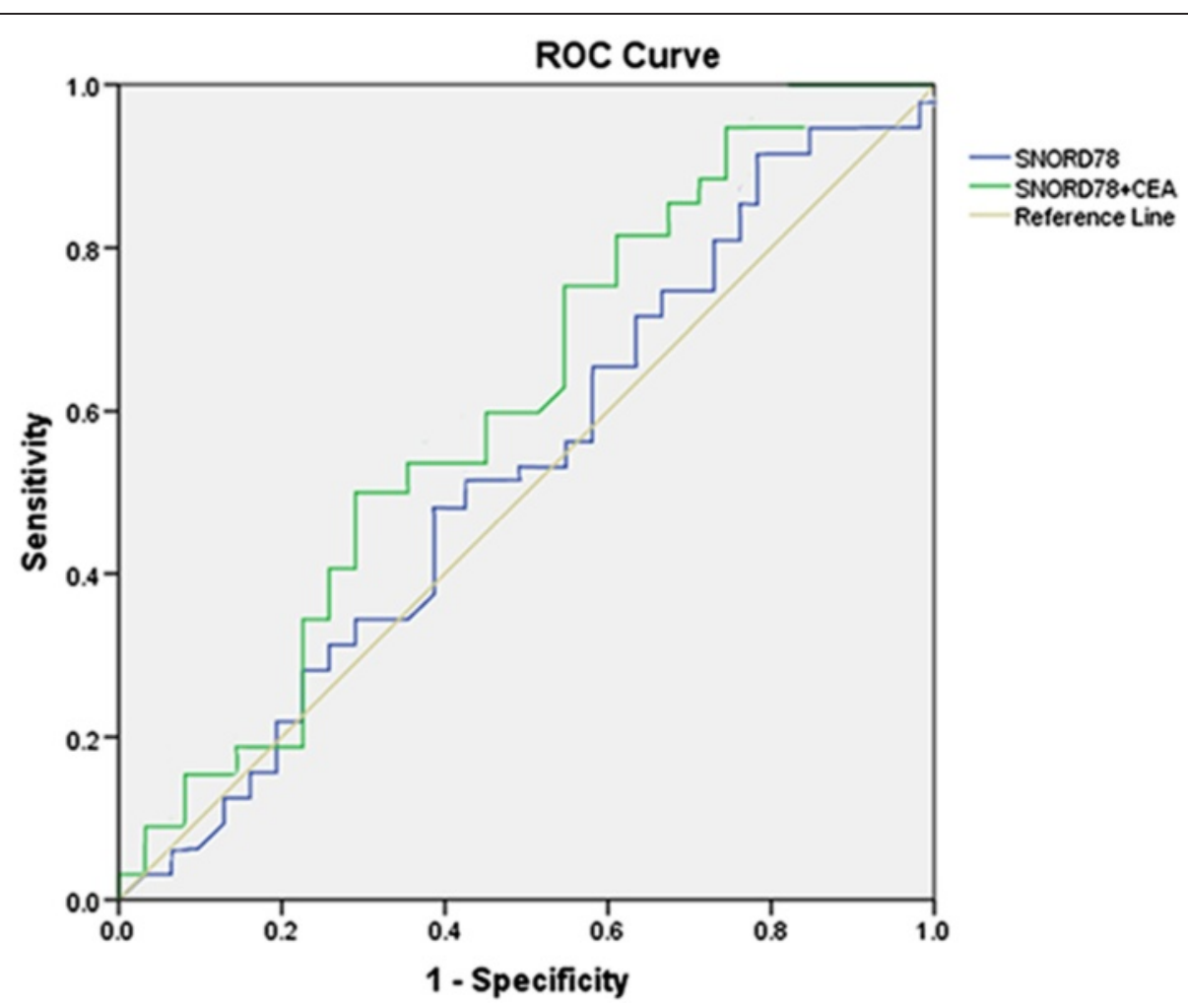

Fig. 8 SNORD78 might be a biomarker in NSCLC. Expression of SNORD78 was detected in subgroups grouped by metastasis. Further ROC curve analysis was used for merged SNORD78 and CEA to predict tumor metastasis in NSCLC 
cell population contains a small population of cells with properties such as self-renewal, multiplex differentiation, chemo- and radio-resistance, high tumorigenicity, and they may play pivotal parts in the development, progression, metastasis, recurrence and multidrug resistance of cancer [12, 13]. The identification of molecules that play a role in the self-renewal of cancer stem-like cells may provide a key standpoint for better understanding tumorigenesis and developing prognostic biomarkers and targeted therapy. As SNORD78 is indeed upregulated in cancer stem-like cells of NSCLC, we knocked down SNORD78 in cancer stemlike cells of lung cancer and found that shRNA-SNORD78 transfected cells formed fewer and smaller mammospheres compared with vector-transfected cells, implying that SNORD78 is important for the self-renewal of cancer stem-like cells of NSCLC. Inhibition of SNORD78 resulted in the downregulation of a series of stemness factors, such as Sox 2 and Oct4, which has been shown to enhance NSCLC malignancy by inducing cancer stem cell-like properties and epithelial-mesenchymal-transition $[25,26]$. The in vivo data complement the in vitro studies of SNORD78 and confirm the oncogenic activity of SNORD78 in NSCLC.

In conclusion, we demonstrate that the expression of SNORD78 was significantly upregulated in NSCLC tissues. We also showed that SNORD78 promoted the proliferation and invasion of NSCLC cells and is vital for the self-renewal of cancer stem-like cells, suggesting that SNORD78 may play a functional role in NSCLC development. Our study may add our understanding to the molecular mechanisms through which SNORD78 contributes to the tumor progression, which may facilitate the development of snoRNA-directed diagnostics and therapeutics against cancers.

\section{Competing interests}

The authors declare that they have no competing interests.

\section{Authors' contributions}

DZ conceived of the study and participated in its design and coordinated and helped to draft the manuscript. DZ, JZ, JN and JL performed the experiments. JYW and LT participated in the design of the study and performed the statistical analysis. LW, BS and GC wrote the paper. All authors read and approved the final manuscript.

\section{Acknowledgements \\ This work was supported by Shanghai Science and Technique Committee (10ZR1424900, 10DJ1400503 and 134119a3200), Shanghai Shenkang Grant SHDC12012111. The funding sources had no role in the study design, in the collection, analysis and interpretation of data; in the writing of the manuscript and in the decision to submit the manuscript for publication.}

\section{Author details}

'Department of Medical Oncology, Shanghai Pulmonary Hospital, Tongji University School of Medicine, 507 Zhengmin Road, Shanghai 200433, People's Republic of China. ${ }^{2}$ Central Lab, Shanghai Pulmonary Hospital, Tongji University School of Medicine, Shanghai, China. ${ }^{3}$ Department of Pathology, Zhongshan Hospital, Fudan University, 180 Fenglin Road, Shanghai 200032, People's Republic of China.
Received: 12 February 2015 Accepted: 8 May 2015

Published online: 15 May 2015

\section{References}

1. Wink KC, Roelofs E, Solberg T, Lin L, Simone 2nd CB, Jakobi A, et al. Particle therapy for non-small cell lung tumors: where do we stand? A systematic review of the literature Front Oncol. 2014;4:292.

2. Leong D, Rai R, Nguyen B, Lee A, Yip D. Advances in adjuvant systemic therapy for non-small-cell lung cancer. World J Clin Oncol. 2014;5(4):633-45.

3. Smith RA1, Manassaram-Baptiste D, Brooks D, Cokkinides V, Doroshenk M, Saslow D, et al. Cancer screening in the United States, 2014: a review of current American Cancer Society guidelines and current issues in cancer screening. CA Cancer J Clin. 2014;64(1):30-51.

4. Verdecchia A, Francisci S, Brenner H, Gatta G, Micheli A, Mangone L, et al. EUROCARE-4 Working Group: Recent cancer survival in Europe: a 2000-02 period analysis of EUROCARE-4 data. Lancet Oncol. 2007;8(9):784-96.

5. Yang Y, Liu L, Zhang Y, Guan H, Wu J, Zhu X, et al. MiR-503 targets PI3K p85 and IKK- $\beta$ and suppresses progression of non-small cell lung cancer. Int J Cancer. 2014;135(7):1531-42.

6. Sun M, Liu XH, Wang KM, Nie FQ, Kong R, Yang JS, et al. Downregulation of BRAF activated non-coding RNA is associated with poor prognosis for non-small cell lung cancer and promotes metastasis by affecting epithelialmesenchymal transition. Mol Cancer. 2014;13:68.

7. Mei YP, Liao JP, Shen J, Yu L, Liu BL, Liu L, et al. Small nucleolar RNA 42 acts as an oncogene in lung tumorigenesis. Oncogene. 2012;31(22):2794-804.

8. Williams GT, Farzaneh F. Are snoRNAs and snoRNA host genes new players in cancer? Nat Rev Cancer. 2012;12(2):84-8.

9. Xu G, Yang F, Ding $C L$, Zhao $L$, Ren $H$, Zhao P, et al. Small nucleolar RNA 113-1 suppresses tumorigenesis in hepatocellular carcinoma. Mol Cancer. 2014;13:216.

10. Lestrade L, Weber MJ. snoRNA-LBME-db, a comprehensive database of human H/ACA and C/D box snoRNAs. Nucleic Acids Res. 2006;34(Database issue):D158-62.

11. Liao J, Yu L, Mei Y, Guarnera M, Shen J, Li R, et al. Small nucleolar RNA signatures as biomarkers for non-small-cell lung cancer. Mol Cancer. 2010;9:198.

12. Geng SQ, Alexandrou AT, Li JJ. Breast cancer stem cells: Multiple capacities in tumor metastasis. Cancer Lett. 2014;349(1):1-7.

13. Abel EV, Simeone DM. Biology and clinical applications of pancreatic cancer stem cells. Gastroenterology. 2013;144:1241-8.

14. Mannello F. Understanding breast cancer stem cell heterogeneity: time to move on to a new research paradigm. BMC Med. 2013;11:169.

15. Mannoor K, Shen J, Liao J, Liu Z, Jiang F. Small nucleolar RNA signatures of lung tumor-initiating cells. Mol Cancer. 2014;13:104.

16. Li R, Wang H, Bekele BN, Yin Z, Caraway NP, Katz RL, et al. Identification of putative oncogenes in lung adenocarcinoma by a comprehensive functional genomic approach. Oncogene. 2006;25(18):2628-35.

17. Jiang F, Yin Z, Caraway NP, Li R, Katz RL. Genomic profiles in stage I primary non small cell lung cancer using comparative genomic hybridization analysis of cDNA microarrays. Neoplasia. 2004;6(5):623-35.

18. Gebhart E. Double minutes, cytogenetic equivalents of gene amplification, in human neoplasia - a review. Clin Transl Oncol. 2005;7(11):477-85.

19. Tam WL, Weinberg RA. The epigenetics of epithelial-mesenchymal plasticity in cancer. Nat Med. 2013;19(11):1438-49.

20. Nieto MA. Epithelial plasticity: a common theme in embryonic and cancer cells. Science. 2013;342(6159):1234850.

21. Rhim AD. Epithelial to mesenchymal transition and the generation of stem-like cells in pancreatic cancer. Pancreatology. 2013;13(2):114-7.

22. Singh S, Trevino J, Bora-Singhal N, Coppola D, Haura E, Altiok S, et al. EGFR/ Src/Akt signaling modulates Sox2 expression and self-renewal of stem-like side-population cells in non-small cell lung cancer. Mol Cancer. 2012;11:73.

23. Chiou SH, Wang ML, Chou YT, Chen CJ, Hong CF, Hsieh WJ, et al. Coexpression of Oct4 and Nanog enhances malignancy in lung adenocarcinoma by inducing cancer stem cell-like properties and epithelialmesenchymal transdifferentiation. Cancer Res. 2010;70(24):10433-44.

24. Kim M, Chen X, Chin LJ, Paranjape T, Speed WC, Kidd KK, et al. Extensive sequence variation in the $3^{\prime}$ untranslated region of the KRAS gene in lung and ovarian cancer cases. Cell Cycle. 2014;3(6):1030-40.

25. Antonicelli A, Cafarotti S, Indini A, Galli A, Russo A, Cesario A, et al. EGFR-targeted therapy for non-small cell lung cancer: focus on EGFR oncogenic mutation. Int J Med Sci. 2013;10(3):320-30. 
26. Wang $L, H u H$, Pan $Y$, Wang $R$, Li $Y$, Shen $L$, et al. PIK3CA mutations frequently coexist with EGFR/KRAS mutations in non-small cell lung cancer and suggest poor prognosis in EGFR/KRAS wildtype subgroup. PLOS One. 2014;9(2), e88291.

27. Yamaguchi F, Kugawa S, Tateno H, Kokubu F, Fukuchi K. Analysis of EGFR, KRAS and P53 mutations in lung cancer using cells in the curette lavage fluid obtained by bronchoscopy. Lung Cancer. 2012;78(3):201-6.

28. Luo J, Tang L, Zhang J, Ni J, Zhang HP, Zhang L, et al. Long non-coding RNA CARLo-5 is a negative prognostic factor and exhibits tumor pro-oncogenic activity in non-small cell lung cancer. Tumour Biol. 2014;35(11):11541-9.

29. Dong XY, Guo P, Boyd J, Sun X, Li Q, Zhou W, et al. Implication of snoRNA U50 in human breast cancer. J Genet Genomics. 2009;36(8):447-54.

30. Dong XY, Rodriguez C, Guo P, Sun X, Talbot JT, Zhou W, et al. SnoRNA U50 is a candidate tumor-suppressor gene at $6 q 14.3$ with a mutation associated with clinically significant prostate cancer. Hum Mol Genet. 2008;17(7):1031-42.

\section{Submit your next manuscript to BioMed Central and take full advantage of:}

- Convenient online submission

- Thorough peer review

- No space constraints or color figure charges

- Immediate publication on acceptance

- Inclusion in PubMed, CAS, Scopus and Google Scholar

- Research which is freely available for redistribution 